\title{
Radio-immunotherapy: a promising weapon to consider in the fight against NSCLC
}

Keywords: treatment, squamous, non-squamous histology, metastatic patients, median survival

Abbreviations: NSCLC, non-small cell lung cancer; CALR, calreticulin; HSPs, heat-shock proteins; APC, antigen-presenting ce1ls; HMGB1, high mobility group box 1; DAMPs, damage-associated molecular patterns; IFN, induced type i interferon; DC-CIK, dendritic cells and cytokine-induced killers

\section{Introduction}

Non-Small Cell Lung Cancer (NSCLC) treatment has dramatically changed during this last decade. Due to better understanding and new options of treatment, unresectable and metastatic patients have improved their overall survival from less than 8 months ${ }^{1}$ to almost 14 months of median survival. ${ }^{2}$ Treatment's algorithm is currently based on the molecular profile of the tumor, especially for lung cancer adenocarcinoma, where the initial study in the metastatic setting includes evaluation of EGFR mutation status, ALK and ROS1 rearrangement, and immunohistochemistry for PDL-1. ${ }^{3}$ The benefit of immunotherapy with PD-1 checkpoint inhibitors has been already reported for squamous and non-squamous histology. 4,5

In the pivotal trial KEYNOTE-024, ${ }^{6}$ pembrolizumab, a humanized monoclonal antibody against PD-1, was associated with an impressive significantly longer progression-free survival in patients with untreated metastatic NSCLC and PD-L1 expression on at least $50 \%$ of tumor cells.

Median progression-free survival was 10.3 months (95\% CI 6.7 to not reached) in the pembrolizumab group versus 6.0 months $(95 \% \mathrm{CI}$, 4.2 to 6.2 ) in the control group (HR $0.50 ; 95 \% \mathrm{CI}, 0.37$ to $0.68 ; \mathrm{P}<0.001$ ). Results for overall survival are not mature yet, but the estimated rate of overall survival at 6 months also favored pembrolizumab. Previous studies ${ }^{4,5}$ reported the benefit of immunotherapy in patients who progressed after a first line of chemotherapy. Although promising results are being reported, the response rate of immunotherapy is still sub-optimal, ${ }^{4-6}$ this could be explained by underlying mechanisms that are currently under study.

Several strategies are under development to improve the published results of immunotherapy, including dual checkpoint blockade with anti-CTLA-4 antibodies and combination with cytotoxic chemotherapy or anti-VEGF antibodies. ${ }^{7}$

The role of fractionated radiotherapy is well established in patients with NSCLC locally advanced disease ${ }^{8}$ and newer technologies, like SBRT, are more frequently used in patients with early stage disease. ${ }^{9}$ Recently the focus has changed due to theoretical advantages that could be obtained with the combination of immunotherapy and radiation therapy in metastatic disease. Although the lymphocytotoxic effect of radiotherapy has long been recognized, ${ }^{10}$ this should be considered with caution, because the newer available technologies allow better control of the irradiation volumes, with decreased filed sizes and optimized dose constraints.
Volume 3 Issue 2 - 2017

\author{
Caglevic Christian, ${ }^{1,2}$ Tudela Benjamin, ${ }^{3}$ \\ Gallardo Carlos E,, ,2 Anabalon Jaime, ,2,3,5,5,6 \\ Panay Sergio, ${ }^{2,3,4,5,6}$ Mahave Mauricio, ${ }^{1,2}$ \\ Marsiglia Hugo 4,5 \\ 'Unit of Investigational Cancer Drugs, Fundacion Arturo Lopez \\ Perez, Chile \\ ${ }^{2}$ Department of Medical Oncology, Fundacion Arturo Lopez \\ Perez, Chile \\ ${ }^{3}$ Radiotherapy Resident, Universidad de Valparaiso, Chile \\ ${ }^{4}$ Medical Director, Fundacion Arturo Lopez Perez, Chile \\ ${ }^{5}$ Department of Radiotherapy, Fundacion Arturo Lopez Perez, \\ Chile \\ ${ }^{6}$ Resident of Medical Oncology, Universidad de Los Andes, Chile
}

Correspondence: Christian Caglevic, Head of Unit of Investigational Cancer Drugs, Fundación Arturo Lopez Perez, Rancagua 878, Providencia, Santiago, Chile, Email oncodemia@yahoo.com, caglevicc@falp.org

Received: June 12, 2017 | Published: June 26, 2017

\section{The rationale of immune-radiotherapy in NSCLC}

Radiation therapy is essentially a localized treatment based on the utilization of ionizing radiation, commonly photons, that damage the DNA, which will cause cell death by different mechanisms. ${ }^{11,12}$ However, effects of radiation are not limited only to the targeted volumes, and tumor regression has been also observed to occur outside the radiation field. This phenomenon is known as "abscopal effect" (ab scopus, away from the target). ${ }^{12,13}$ The complex mechanism behind this phenomenon is not completely understood, but immunemediated cell death is thought to be a main component of the event. When the irradiated cancerous cell dies, it releases damage-associated molecular patterns (DAMPs) which recruit cellular components of the immune system to the tumor bed, creating an anti-tumor immune response. ${ }^{13}$ Some of these molecules include the endoplasmic reticulum chaperone calreticulin (CALR) and various heat-shock proteins (HSPs), which are exposed on the surface of the dying cell and recognized by antigen-presenting cells (APC). Also, these proteins can be released as cytokines. ${ }^{14}$ The nuclear High Mobility Group Box 1 protein (HMGB1) and ATP are also released from the dying cell and recognized by de APC. ${ }^{12}$ The final effects of these pathways lead to an improved uptake of tumor cells and recognition of tumor-derived antigens. Other effects provoked by radiation include increased expression of NKG2D ligands and sensitivity to NK cell-mediated cytotoxicity of tumor cells ${ }^{15}$ and radiation-induced type I interferon (IFN) production, upregulated MHC class I expression, and restored response to anti-PD-1 in anti-PD-1-resistant rat models. ${ }^{16}$ These molecular mechanisms open the possibility of a synergistic effect when used in conjunction with immunotherapy. 
The production of tumor-derived antigens is associated with the mutational heterogeneity of cancer cell lines, some types of cancers such as NSCLC present high somatic mutation frequency, which is thought to be associated with exposure to carcinogens like tobacco. ${ }^{17}$ Although the immune system can detect tumor-derived antigens, there are mechanisms to evade immune-mediated destruction of cancer cells, like down-regulation of major histocompatibility complex and expression of cell surface proteins that kill cytotoxic $\mathrm{T}$ cell (PD-L1-PD-1 axis) among others ${ }^{18}$ which can be manipulated via immunotherapy and radiation therapy. NSCLC cells express PD-L1 as a mechanism to avoid immune response, and it has been used in the clinic as a biomarker for efficacy of immunotherapy, identifying better response rates to anti-PD-1 immunotherapy in patients with higher cellular membrane expression. ${ }^{19}$ Higher somatic nonsynonymous mutation burden and molecular signature of smoking have also shown association with clinical response to anti-PD1 immunotherapy. ${ }^{20}$

\section{Clinical evidence of the benefit of combined treatment}

Currently, most of the evidence that supports the use of immunotherapy combined with radiation therapy comes from preclinical models. In the clinical setting, the incidence of abscopal effect in patients receiving immunotherapy has been reported more frequently in melanoma. ${ }^{21}$ In NSCLC patients some case reports have been published, creating interest in the combination of ablative radiation therapy and immunotherapy. Golden et al., ${ }^{22}$ reported a case of a 64years-old man with metastatic lung adenocarcinoma who received treatment with IMRT to a total dose of 30Gy to a liver metastasis concurrently with ipilimumab $3 \mathrm{mg} / \mathrm{kg}$ body weight. Posttreatment imaging showed response inside and outside the irradiated field. Cong et al. ${ }^{23}$ reported the case of a 64year-old- treated female with metastatic lung adenosquamous carcinoma. After progression, she was treated with dendritic cells and cytokine-induced killers (DCCIK) immunotherapy and concurrent SBRT to a dose of $37.5 \mathrm{~Gy}$ in five fractions to a paramediastinal lesion. Chest imaging performed 10months after completion of SBRT showed response of lesions in the irradiated field and outside of it as well.

A recently published retrospective analysis of the KEYNOTE-001 trial, ${ }^{24}$ reported the outcomes in patients previously treated with radiotherapy. Progression-free survival and overall survival was significantly improved for patients treated with pembrolizumab who received previous radiotherapy compared with those without a history of radiotherapy (HR $0.5695 \% \mathrm{CI}, 0.34-0.91 ; \mathrm{P}=0.019$ and HR 0.58; 95\%CI, 0.36-0.94; $\mathrm{P}=0.026$, respectively).

To date, several clinical trials are being conducted to further clarify the effects of this approach in patients with localized and metastatic $\operatorname{NSCLC}(25,26)$. From the oncological perspective, several questions must be answered in the clinical field, including optimal time of delivery of radiation, dose and fractionation and particle employed to optimize outcomes but also how to combine radiotherapy and immunotherapy looking forward to achieve the best results in survival with a lesser toxicity rate.

\section{Conclusion}

Immunotherapy has become a promising treatment option for patients with metastatic NSCLC but evidence suggests that there is still a wide range of opportunities to further improve outcomes. While pre-clinical studies are providing valuable information, more clinical research is required to confirm the efficacy of radio-immunotherapy in
NSCLC patients. The abscopal effect can be considered as a promising weapon in the fight against NSCLC in patients with metastatic and unresectable disease that needs to be studied and better explored in prospective randomized clinical trials.

\section{Acknowledgements}

None.

\section{Conflict of interest}

Author declares that there is no conflict of interest.

\section{References}

1. Schiller JH, Harrington D, Belani CP, et al. Comparison of Four Chemotherapy Regimens for Advanced Non-Small-Cell Lung Cancer. $N$ Engl J Med. 2002;346(2):92-98.

2. Paz-Ares LG, de Marinis F, Dediu M, et al. PARAMOUNT: final overall survival results of the phase III study of maintenance pemetrexed versus placebo immediately after induction treatment with pemetrexed plus cisplatin for advanced nonsquamous non-small-cell lung cancer. J Clin Oncol. 2013;31(23):2895-2902.

3. https://www.nccn.org/professionals/physician_gls/PDF/nscl.pdf

4. Brahmer J, Reckamp KL, Baas P, et al. Nivolumab versus docetaxel in advanced squamous-cell non-small-cell lung cancer. $N$ Engl J Med. 2015;373(17):123-135.

5. Borghaei H, Paz-Ares L, Horn L, et al. Nivolumab versus docetaxel in advanced nonsquamous non-small-cell lung cancer. $N$ Engl J Med. 2015;373:1627-1639.

6. Reck M, Rodríguez-Abreu D, Robinson AG, et al. KEYNOTE-024 Investigators, et al. Pembrolizumab versus chemotherapy for PD-L1-positive non-small-cell lung cancer. NEngl J Med. 2016;375(19):1823-1833.

7. Remon J, Besse B, Soria JC. Successes and failures: what did we learn from recent first-line treatment immunotherapy trials in non-small cell lung cancer? BMC Med. 2017;15(1):55.

8. Albain KS, Swann RS, Rusch VW, et al. Radiotherapy plus chemotherapy with or without surgical resection for stage III non-smallcell lung cancer: a phase III randomised controlled trial. Lancet. 2009;374(9687):379-386.

9. Siva S, Ball D. Curing Operable Stage I Non-Small Cell Lung Cancer With Stereotactic Ablative Body Radiotherapy: The Force Awakens. Oncologist. 2016;21(4):393-398.

10. Order SE. The effects of therapeutic irradiation on lymphocytes and immunity. Cancer. 1977;39(2):737-743.

11. Eriksson D, Stigbrand T. Radiation-induced cell death mechanisms. Tumour Biol. 2010;31(4):363-372.

12. Golden EB, Pellicciotta I, Demaria S, et al. The convergence of radiation and immunogenic cell death signaling pathways. Front Oncol. 2012;2:88

13. Reynders K, Illidge T, Siva S, et al. The abscopal effect of local radiotherapy: using immunotherapy to make a rare event clinically relevant. Cancer Treat Rev. 2015;41(6):503-510.

14. Fucikova J, Moserova I, Urbanova L, et al. Prognostic and Predictive Value of DAMPs and DAMP-Associated Processes in Cancer. Front Immunol. 2015;6:402.

15. Kim JY, Son YO, Park SW, et al. Increase of NKG2D ligands and sensitivity to NK cell-mediated cytotoxicity of tumor cells by heat shock and ionizing radiation. Exp Mol Med. 2006;38(5):474-484. 
16. Wang X, Schoenhals JE, Li A, et al. Suppression of type I IFN signaling in tumors mediates resistance to anti-PD-1 treatment that can be overcome by radiotherapy. Cancer Res. 2017;77(4):839-850.

17. Lawrence MS, Stojanov P, Polak P, et al. Mutational heterogeneity in cancer and the search for new cancer genes. Nature. 2013;499(7457):214-218.

18. Naing A, Hajjar J. Immunotherapy. 1st ed. Switzerland: Springer International Publishing; 2017. p. 97-125.

19. Garon EB, Rizvi NA, Hui R, et al. Pembrolizumab for the treatment of non-small-cell lung cancer. N Engl J Med. 2015;372(21):2018-2028.

20. Rizvi NA, Hellmann MD, Snyder A, et al. Mutational landscape determines sensitivity to PD-1 blockade in non-small cell lung cancer. Science. 2015;348(6230):124-128.

21. Ribeiro Gomes J, Schmerling RA, Haddad CK, et al. Analysis of the abscopal effect with anti-PD1 therapy in patients with metastatic solid tumors. J Immunother. 2016;39(9):367-372.
22. Golden EB, Demaria S, Schiff PB, et al. An Abscopal Response to Radiation and Ipilimumab in a Patient with Metastatic Non-Small Cell Lung Cancer. Cancer Immunol Res. 2013;1(6):365-372.

23. Yang Cong, Ge Shen, Shikai Wu, et al. Abscopal regression following SABR for non-small-cell-lung cancer: A case report. Cancer Biol Ther. 2017;18(1):1-3

24. Shaverdian N, Lisberg AE, Bornazyan K, et al. Previous radiotherapy and the clinical activity and toxicity of pembrolizumab in the treatment of non-small-cell lung cancer: a secondary analysis of the KEYNOTE-001 phase 1 trial. The Lancet Oncology. 2017.

25. Hanna GG, Illidge T. Radiotherapy and Immunotherapy Combinations in Non-small Cell Lung Cancer: A Promising Future? Clin Oncol. 2016;28(11):726-731

26. Daly ME, Monjazeb AM, Kelly K. Clinical trials integrating immunotherapy and radiation for non-small-cell lung cancer. $J$ Thorac Oncol. 2015;10(12):1685-1693. 